\title{
Distinct Inflammatory Changes of the Pancreas of Slowly Progressive Insulin-dependent (Type 1) Diabetes
}

\section{SUPPLEMENTAL DIGITAL CONTENT}

SUPPLEMENTAL TABLE 1. HLA-DR, Frequencies of Pseudoatrophic (Insulin-deficient) Islets With Insulitis and PanIN Grade in SPIDDM

\begin{tabular}{lcccc}
\hline & & \multicolumn{2}{c}{ Pseudoatrophic Islet } & \\
\cline { 3 - 4 } Case Subject & HLA-DR & Frequencies, (\%) & $\begin{array}{c}\text { Insulin-deficient Islet } \\
\text { With Insulitis, (\%) }\end{array}$ & PanIN Grade \\
\hline SPIDDM & & & & \\
SP-1 & $4 / 9$ & $3 / 38(7.9)$ & $1 / 3(33.3)$ & PanIN-1A \\
SP-2 & ND & $0 / 36(0.0)$ & $0 / 0(0.0)$ & PanIN-2 \\
SP-3 & ND & $0 / 40(0.0)$ & $0 / 0(0.0)$ & PanIN-2 \\
SP-4 & ND & $0 / 40(0.0)$ & $0 / 0(0.0)$ & PanIN-1A \\
SP-5 & $4 /-$ & $8 / 48(16.7)$ & $3 / 8(37.5)$ & - \\
SP-6 & $9 / 13$ & $84 / 84(100)$ & $14 / 84(16.7)$ & PanIN-1A \\
SP-7 & $4 /-$ & $29 / 29(100)$ & $1 / 29(3.5)$ & - \\
SP-8 & $4 / 9$ & $0 / 40(0.0)$ & $0 / 0(0.0)$ & PanIN-2 \\
SP-9 & $9 /-$ & $31 / 31(100)$ & $5 / 31(16.1)$ & PanIN-2 \\
SP-10 & $4 / 8$ & $1 / 13(7.7)$ & $0 / 1(0.0)$ & - \\
SP-11 & $4 / 6$ & $4 / 67(6.0)$ & $0 / 4(0.0)$ & PanIN-1A \\
SP-12 & $4 / 9$ & $2 / 59(3.4)$ & $0 / 2(0.0)$ & PanIN-2 \\
\hline
\end{tabular}

PanIN indicates pancreatic intraepithelial neoplasia; ND, not detected. 